\title{
Time-reversal symmetric topological metal
}

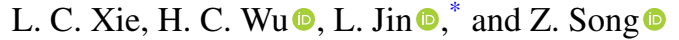 \\ School of Physics, Nankai University, Tianjin 300071, China
}

(Received 8 June 2021; revised 14 September 2021; accepted 18 October 2021; published 25 October 2021)

\begin{abstract}
Topological metals possess gapless band structures accompanied by nontrivial edge states. Topological metals are created from the topological insulators by adjusting the magnetic flux. Here we propose the time-reversal symmetry protected topological metal without employing the magnetic flux. Topological metallic phase presents although the Chern number vanishes. In the topologically nontrivial phase, three different cases in the metallic phase are distinguished from the band-touching and in-gap features of the topological edge states. These findings shed light on the time-reversal symmetric topological metals and greatly simplify the realization of topological metals.
\end{abstract}

DOI: 10.1103/PhysRevB.104.165422

\section{INTRODUCTION}

The introduction of topology in condensed matter physics greatly changes the traditional way of classifying phases of matter, novel topological states including insulators, superconductors, semimetals, and metals are discovered [1-14] . These topological phases all support robust edge states, which are counted by the corresponding topological invariant that captures the topology of the bulk band [15-17]. The tremendous progresses in various platforms of photonics, acoustics, and electronic circuits allow us to study these topological phases in experiments, which provide crucial enlightenments for topological materials [18-30] .

Topological insulators have a fully gapped bulk band structure like ordinary insulators, but topological insulators support conductive edge states on the surface of the sample, where electrons move along the surface of materials [2]. The Chern insulator is a typical two-dimensional (2D) topological insulator, which supports robust chiral edge states that are predicted from the nonzero Chern number. A landmark development of topological insulators is the discovery of time-reversal invariant topological insulators [31-34], where the Chern number vanishes and becomes invalid for topological characterization; alternatively, the $Z_{2}$ topological number classifies the time-reversal invariant insulators [35] and these topological insulators are featured by their helical edge states, being confirmed in a nonlocal transport experiment [36]. The helical edge states have opposite spins and counterpropagate on the boundaries in the opposite directions. The spin Chern number [37-39], valley Chern number [40-42], and wave polarization

\footnotetext{
*jinliang@nankai.edu.cn
}

Published by the American Physical Society under the terms of the Creative Commons Attribution 4.0 International license. Further distribution of this work must maintain attribution to the author(s) and the published article's title, journal citation, and DOI.
[43-45] are developed to characterize the quantum spin Hall effect.

Topological semimetals and metals are metallic states with nontrivial gapless band structure and unique quasiparticle excitations [8], represented by Weyl and Dirac semimetals. In the Weyl (Dirac) semimetals, energy bands are double (fourfold) degenerately connected by the Weyl (Dirac) points [46-48]. In addition, the existence of other multiple degeneracy points have been discovered, like threefold and sixfold [49-54]. For systems protected by certain symmetries, the band connection is a closed node line instead of nodes. The corresponding topological node-line semimetals have been reported in the graphene network [55-57].

Topological metals (TMs) are gapless phases featured by their separable conduction and valence bands. Thus, the topological characterization for the metals is similar as that for the insulators. TMs can be divided into nodal-point, nodal-line, and nodal-surface TMs according to the dimension of band crossing [58]; or alternatively be divided into type I and type II TMs according to the dispersion close to the band crossing [59-61]. TMs in different spatial dimensions have been extensively studied [62-64]. To create a 2D topological metal, the traditional method is through adjusting the magnetic flux to tune the energy bands of the topological insulators [65]. However, introducing and tuning the magnetic flux is difficult in many experimental platforms. Here we demonstrate a timereversal symmetric topological metal without the magnetic flux.

In this paper we report the time-reversal symmetric topological metal in a 2D square lattice, which belongs to class AI in the tenfold way of topological classification. The 2D square lattice possesses nontrivial topology although the Chern number is zero ensured by the time-reversal symmetry. The proposed topological metal is free from the magnetic flux and significantly differs from the traditional topological metal created through using the magnetic flux. The reflection symmetry ensures the quantized polarization; the zero polarization predicts the trivial topology in the vertical direction. The Zak phase at the phase transition point characterizes the 


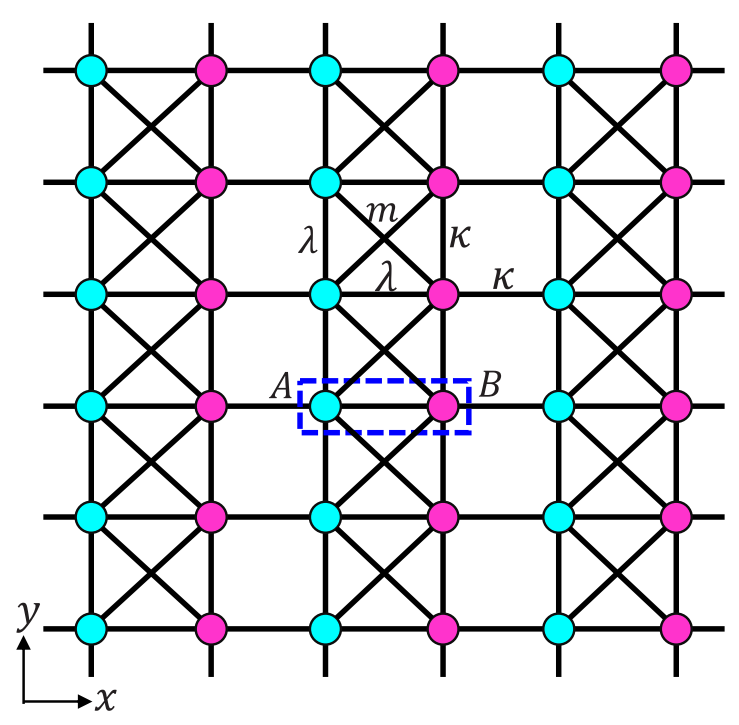

FIG. 1. Schematic of the 2D square lattice. The unit cell consists of two sublattices $A$ and $B$ in cyan and magenta as indicated inside the dashed blue rectangle. $\lambda, \kappa$, and $m$ are the couplings between the sublattices.

topology in the horizontal direction. We demonstrate three different cases in the topologically nontrivial phases that are distinguished from their different types of topological edge states. The topological phases as well as the topological edge states can be deformed into each other without undergoing topological phase transition. Our findings provide novel insights into time-reversal symmetric topological metals.

The remainder of the paper is organized as follows. In Sec. II the time-reversal symmetric 2D square lattice is introduced. Section III presents the phase diagram and the energy band of the topological metallic phase. Three different topological edge states are elucidated. Section IV provides the topological characterization. The discussion and conclusion are summarized in Secs. V and VI.

\section{TIME-REVERSAL SYMMETRIC 2D SQUARE LATTICE}

We consider a time-reversal symmetric 2D square lattice. The schematic of the lattice is illustrated in Fig. 1. The Hamiltonian of the 2D square lattice in the real space is written in the form of

$$
\begin{aligned}
H= & \sum_{l, j}\left(\lambda a_{l, j}^{\dagger} b_{l, j}+\kappa a_{l, j}^{\dagger} b_{l-1, j}+m a_{l, j}^{\dagger} b_{l, j+1}\right. \\
& \left.+m a_{l, j}^{\dagger} b_{l, j-1}+\text { H.c. }\right)+\lambda a_{l, j}^{\dagger}\left(a_{l, j+1}+a_{l, j-1}\right) \\
& +\kappa b_{l, j}^{\dagger}\left(b_{l, j+1}+b_{l, j-1}\right),
\end{aligned}
$$

where $a^{\dagger}, b^{\dagger}(a, b)$ represent the creation (annihilation) operators for the sublattices $A$ and $B$. The dashed box in blue indicates the unit cell of the system. The couplings $\lambda$ and $\kappa$ indicate the nearest neighbor coupling strengths, the coupling $m$ is the next nearest neighbor coupling strength. The 2D square lattice does not enclose any magnetic flux and can be realized in many versatile experimental platforms for the topological insulators including the ultracold atoms in the optical lattice, the photonic crystals of coupled optical waveguides/resonators, the acoustic metamaterial dubbed sonic crystals, and the electric circuits [66-72].

Applying the Fourier transformation $\rho_{l, j}=$ $M^{-1} \sum_{\mathbf{k}} e^{i \mathbf{k} \cdot \mathbf{r}} \rho_{\mathbf{k}}(\rho=a, b)$ to the two sublattices, we rewrite the lattice Hamiltonian $H$ in the momentum space in the form of

$$
H=\sum_{\mathbf{k}} H(\mathbf{k})=\sum_{\mathbf{k}} \psi_{\mathbf{k}}^{\dagger} h(\mathbf{k}) \psi_{\mathbf{k}},
$$

where the basis is $\psi_{\mathbf{k}}=\left(a_{\mathbf{k}}, b_{\mathbf{k}}\right)^{T}$ and the Bloch Hamiltonian $h(\mathbf{k})$ is a $2 \times 2$ matrix

$$
h(\mathbf{k})=d_{0} \sigma_{0}+\mathbf{d} \cdot \sigma,
$$

where $\sigma_{0}$ is the $2 \times 2$ identical matrix, $d_{0}=(\lambda+\kappa) \cos k_{y}$. $\sigma=\left(\sigma_{x}, \sigma_{y}, \sigma_{z}\right)$ is the Pauli matrices, and the effective magnetic field $\mathbf{d}$ is

$$
\begin{aligned}
& d_{x}=\lambda+2 m \cos k_{y}+\kappa \cos k_{x}, \\
& d_{y}=\kappa \sin k_{x}, \\
& d_{z}=(\lambda-\kappa) \cos k_{y} .
\end{aligned}
$$

The energies of the Bloch bands are

$$
E_{ \pm}=d_{0} \pm \sqrt{d_{x}^{2}+d_{y}^{2}+d_{z}^{2}}
$$

where the subscripts + and - of energy represent the upper and lower bands, respectively. Without loss of generality, we fix the coupling $m$ as unity. Notably, the band gap closes at $\lambda= \pm \kappa$, associated with the appearance of degenerate points (DPs) or degenerate lines.

The Bloch Hamiltonian $h(\mathbf{k})$ of the square lattice is protected by the time-reversal symmetry. Thus, the band energies satisfy $E_{ \pm, \mathbf{k}}=E_{ \pm,-\mathbf{k}}^{*}$ as reflected from Eq. (5). The symmetries are important $[11,13,73]$. Notably, the time-reversal symmetry ensures a zero Chern number for the energy band; however, the topologically nontrivial phase still exists in the 2D lattice $[45,74]$. $d_{0}$ adjusts the energy band and changes the band structure, and plays a crucial role in creating the metallic phase when the strengths of the couplings $\lambda$ and $\kappa$ are not equal $\lambda^{2} \neq \kappa^{2}$. In the absence of $d_{0}$, the bands are fully gapped except for the band touching and the system is in an insulator phase.

The energy bands have highly degenerate points, where the energy bands become fully flat. The highly degenerate points appear at $k_{y}= \pm \arccos (-\lambda / 2)$, but disappear as $|\lambda|>$ 2 . Time-reversal symmetry ensures that the two highly degenerate points are symmetric about $k_{y}=0$. The upper and lower bands reduce to two momentum independent flat bands

$$
\varepsilon_{ \pm}=-\left(\lambda^{2}+\kappa \lambda \pm \sqrt{\Delta}\right) / 2
$$

where $\Delta=4 \kappa^{2}+\kappa^{2} \lambda^{2}-2 \kappa \lambda^{3}+\lambda^{4}$. Notice that the presence of $d_{0}$ breaks the symmetry of the upper and lower bands about zero energy.

We emphasize that the proposed topological metal significantly differs from the traditional topological metal created through altering the magnetic flux. Their symmetry protections, topological classifications, and topological invariants are different. The proposed topological metal without magnetic flux is time-reversal symmetric and belongs to the symmetry class AI [75]. In contrast, the topological metal with 


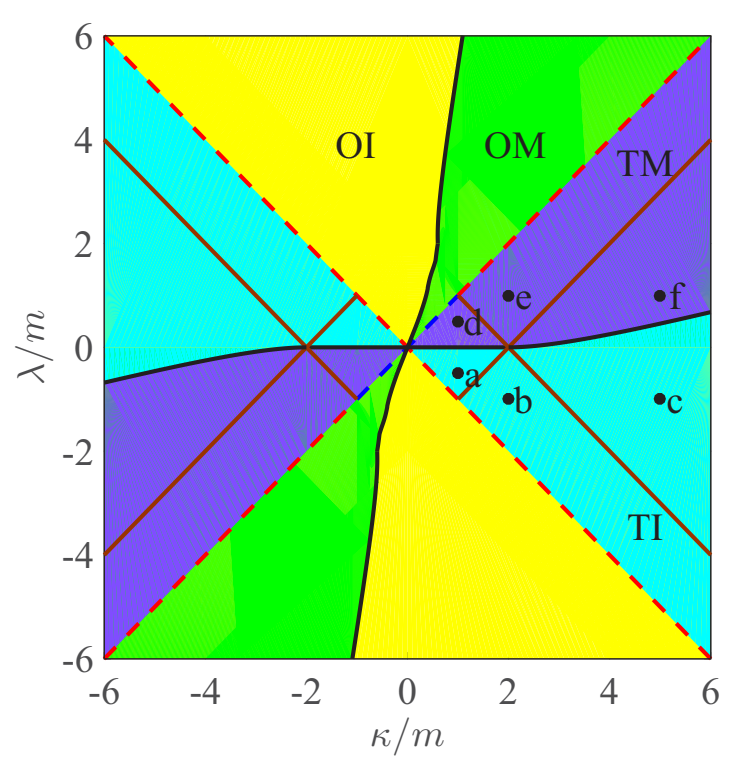

FIG. 2. Phase diagram of $h(\mathbf{k})$ in the $\kappa-\lambda$ parameter space. Regions with different colors indicate different phases: topological metal (TM) in purple, ordinary metal $(\mathrm{OM})$ in green, topological insulator (TI) in cyan, and ordinary insulator (OI) in yellow. The black solid lines indicate the Wilson transitions. The red dashed lines indicate the topological phase transition with two DPs, while the blue dashed line indicates the topological phase transition with four DPs. The brown solid lines are the boundary that characterizes the positional relationship between the edge states and bulk bands, indicating three different cases in the topological metallic or insulator phase.

magnetic flux breaks the time-reversal symmetry; for example, the topological metal in Ref. [65] created via tuning the magnetic flux from the ordinary metal is particle-hole symmetric and belongs to the symmetry class D. Consequently, the topological invariants are different. In addition, the energy bands are symmetric about $k_{y}=0$ in the time-reversal symmetric topological metal. Thus, the edge states cross twice at zero energy as a consequence of zero Chern number $C=0$; and the dispersions and velocities near the zero edge states on the two sides of $k_{y}=0$ are opposite. We can observe these features in the following section. However, the edge states cross once at zero energy in the traditional topological metal without the time-reversal symmetry protection as a consequence of nonzero Chern number $C= \pm 1$.

\section{METAL-INSULATOR TRANSITION}

In this section we discuss the topological phases. The prominent feature of the considered 2D square lattice is the presence of topological metallic phase. For a two-band model, if the bulk bands are separable and the Fermi level lies in the band gap, the system is an insulator. Once the highest energy level of the valence band exceeds the lowest energy level of the conduction band, the two band energies partly overlap each other and the Fermi level crosses the two bands. Then, the system completes the transition from insulator to metal, known as the Wilson transition. Figure 2 is the phase diagram, being inversion invariant about the origin $(\kappa, \lambda)=(0,0)$. The
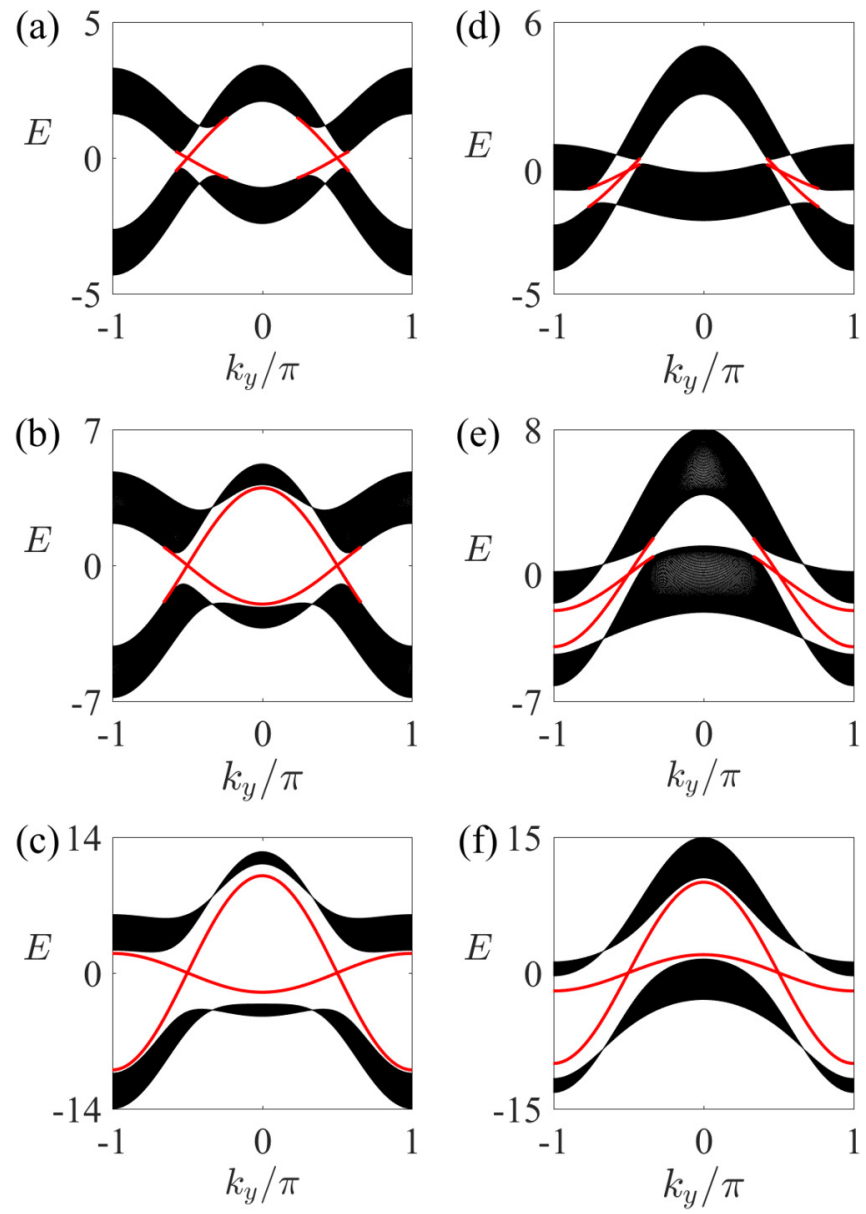

FIG. 3. Band structures of three cases in the insulator phase at (a) $\kappa=1, \lambda=-0.5$, (b) $\kappa=2, \lambda=-1$, (c) $\kappa=5, \lambda=-1$ and three cases in the metallic phase at (d) $\kappa=1, \lambda=0.5$, (e) $\kappa=$ $2, \lambda=1$, (f) $\kappa=5, \lambda=1$ as indicated by the black solid dots in the phase diagram of Fig. 2. The energy band is depicted for the OBC in the $x$ direction and the $\mathrm{PBC}$ in the $y$ direction. The other system parameter is $m=1$. The red line indicates the edge state.

dashed lines represent the topological phase transition, and the solid black lines represent the Wilson transition. The purple and cyan regions are the metallic and insulator phases, respectively; being the topologically nontrivial phases. Similarly, the green and yellow regions are the metallic and insulator phases, respectively; being the trivial phases.

The topological insulator phase is indicated by the cyan region in the phase diagram Fig. 2. The two bands are fully gapped as shown in the energy spectra Figs. 3(a)-3(c). The topological metallic phase is indicated by the purple region in the phase diagram Fig. 2. The lowest energy of the conduction band is lower than the highest energy of the valence band as shown in the energy spectra Figs. 3(d)-3(f). When the system in the insulator phase crosses the solid black lines in the phase diagram Fig. 2, the system undergoes a Wilson phase transition and enters the metallic phase. However, the band gap is always open, which ensures that the topological characteristics remain unchanged. This indicates that the topological metallic phase and the topological insulator phase have identical topological properties. 
Interestingly, the system has three different types of edge states featured from the relation between the edge states and the bulk bands in both the topological metallic phase and the topological insulator phase. The edge states always intersect at the momentum $k_{y}= \pm \pi / 2$, which is also the band closing point. These are observed from the energy spectra of Figs. 3(a)-3(c) and Figs. 3(d)-3(f), respectively. The different types of edge states can be deformed into each other without the band gap closing; thus, they possess identical topological properties. These edge states are distinguished by the lines $\lambda+\kappa \pm 2=0, \lambda-\kappa \pm 2=0$ as the solid brown lines in the phase diagram of Fig. 2. The topological insulator phase in the $\kappa>0$ is divided into three regions; the spectra for points $a, b$, and $c$ are representative cases. In Fig. 3(a) the system has two pairs of gapless edge states, connecting the upper and the lower bulk bands. In Fig. 3(b) the gapless edge states near the center of the Brillouin zone (BZ) detach the bulk bands, forming two edge states cross twice at $k_{y}= \pm \pi / 2$ with each edge state connected to one bulk band, respectively. In Fig. 3(c) the edge states are completely detached from the bulk bands and become the in-gap edge states. The edge states in the topological metallic phase inherit the characteristics of the edge states in the topological insulator phase. Figures 3(d) to 3(f) show the three typical energy spectra for the topological metallic phase. The topological metal still has separable energy bands. In Fig. 3(d) the gapless edge states intact the bulk bands. In Fig. 3(e) the edge states detach the bulk bands near the boundary of the BZ. In Fig. 3(f) the edge states are completely detached from the bulk band and become in-gap edge states.

For the three cases of insulator or metal as separated by the brown solid lines in Fig. 2, we introduce the total inverse participation ratio (TIPR) as a criterion to distinguish their different features. The existence of edge states can be measured by the quantity of TIPR, which is defined as

$$
\operatorname{TIPR}\left(k_{y}\right)=\sum_{n, l}\left|\left\langle l \mid \psi_{n, k_{y}}\right\rangle\right|^{4},
$$

where $n$ is the band index and $\left|\psi_{n, k_{y}}\right\rangle$ is eigenstate. In the large size limit, the value of $\operatorname{TIPR}\left(k_{y}\right)$ is stable. The distribution of TIPR has three different configurations. The TIPR $\left(k_{y}\right)$ has relative large values in two separated regions, in single incomplete region, and in the whole region of the Brillouin zone of $k_{y}$. The TIPR $\left(k_{y}\right)$ in the topological insulator phase is shown in Figs. 4(a)-4(c) and the TIPR $\left(k_{y}\right)$ in the topological metal phase is shown in Figs. 4(d)-4(f). The distribution can be characterized by the scattering process, where the midgap levels are detected. Each region opens a window in the momentum space (or energy) for the channel of electron transport, and then can be detected by measuring the edge electrical conductivity and thermal conductivity [76].

The insulator phase and metallic phase can be distinguished from the local density of states (LDOS), which describes a space-resolved density of states. The LDOS at the position $x$ and the energy $E$ is defined as $D(E, x)=$ $\sum_{n}\left|\psi_{n}(x)\right|^{2} \delta\left(E-\varepsilon_{n}\right)$ in a continuous system, and the LDOS reduces to the form

$$
D(E, l)=\frac{1}{\chi} \sum_{n=1}^{N} e^{-\alpha^{2}\left(E_{n}-E\right)^{2}}\left|\left\langle l \mid \psi_{n}\right\rangle\right|^{2}
$$
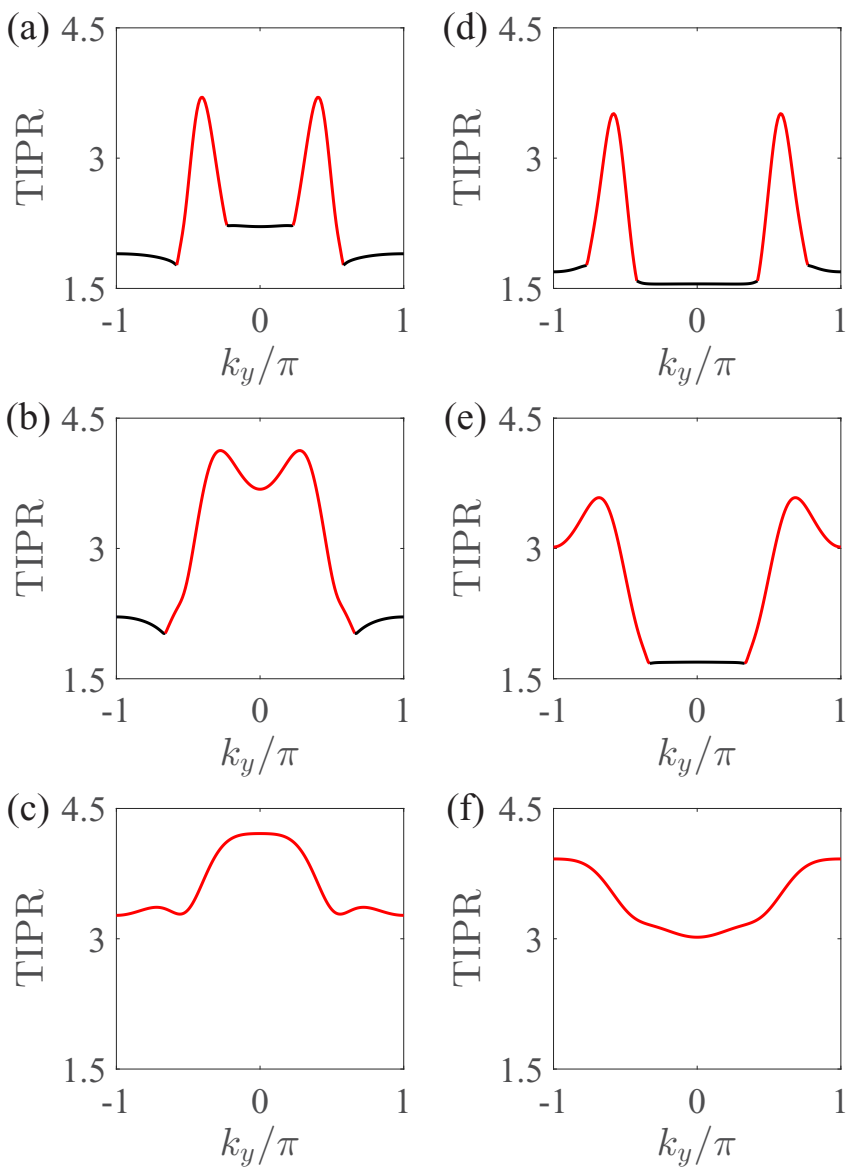

FIG. 4. Plot of the total inverse participation ratio along the momentum $k_{y}$ as the counterpart of Fig. 3. The red line represents the TIPR including the localized edge state, and the black line represents the TIPR of extended states in bulk. (a) $\kappa=1, \lambda=-0.5$, (b) $\kappa=$ $2, \lambda=-1$, (c) $\kappa=5, \lambda=-1$, (d) $\kappa=1, \lambda=0.5$, (e) $\kappa=2, \lambda=1$, (f) $\kappa=5, \lambda=1$.

at the lattice site $l$ and the energy $E$ in a discrete system. In Eq. (8) the delta function is replaced by a Gaussian function and $\chi$ is the renormalization factor. Here we consider the half-filling situation. The LDOS at the Fermi surface $E_{F}=0$ is calculated for the finite size systems with several representative parameters; the results are shown in Fig. 5. $D(E, l)$ has vanishing distributions in all the insulating phases as shown in Figs. 5(a)-5(c), but has finite distributions in all the conducting phases as shown in Figs. 5(d)-5(f). In addition, $D(E, l)$ has evidently large distributions along only two sides in all the phases, resulting in the relatively large electrical and thermal conductivities. The LDOS is detected by a scanning tunneling microscope (STM), which is capable of imaging electron densities of states with atomic resolution [77]. The TIPR and LDOS are measurable quantities in experiment. Furthermore, the nonzero bulk current of the topological metal differs from that of the insulator and the relatively larger edge current of the topological metal differs from that of the normal conductor.

In the topological phases, the edge states exist for the system under the periodic boundary condition (PBC) in the $y$ direction and the open boundary condition (OBC) in the $x$ 
(a)

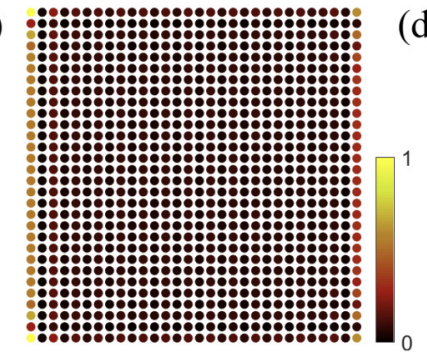

$x$

(b)

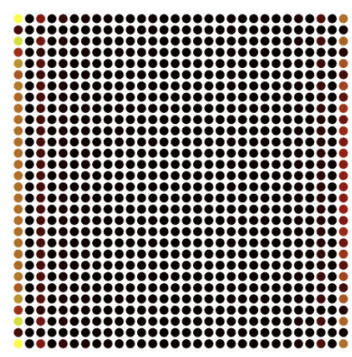

(c)

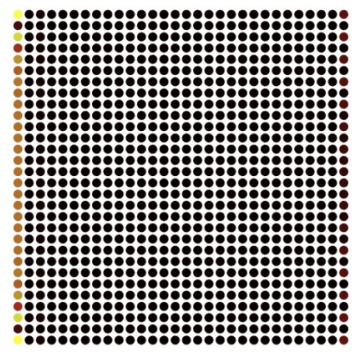

(d)

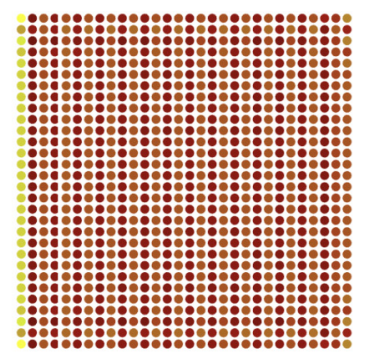

(e)

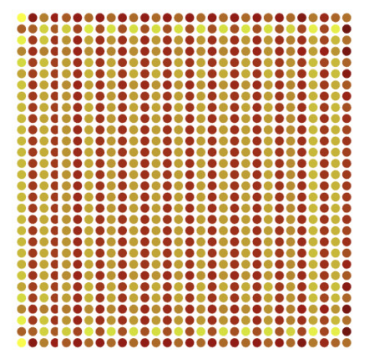

(f)

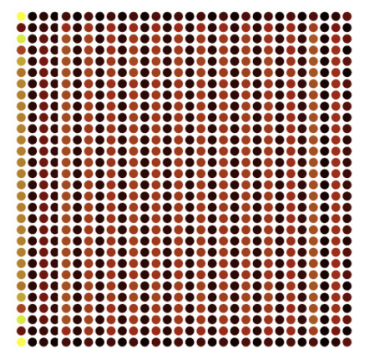

FIG. 5. Plot of the local density of state near the zero energy as the counterpart of Fig. 3. (a) $\kappa=1, \lambda=-0.5$, (b) $\kappa=2, \lambda=-1$, (c) $\kappa=5, \lambda=-1$, (d) $\kappa=1, \lambda=0.5$, (e) $\kappa=2, \lambda=1$, (f) $\kappa=$ $5, \lambda=1$. Here we take $\alpha=2$ and lattice size is $30 \times 30$. The color bar indicates the probability of wave function and the maximum is renormalized to 1 .

direction. The energies of the edge states that localized on the left and right boundaries are

$$
E_{L}\left(k_{y}\right)=2 \lambda \cos k_{y}, \quad E_{R}\left(k_{y}\right)=2 \kappa \cos k_{y} .
$$

The wave function for the edge state with $E_{L}\left(k_{y}\right)\left[E_{R}\left(k_{y}\right)\right]$ localized at the left (right) boundary is denoted as $\left|\psi_{L}\right\rangle\left(\left|\psi_{R}\right\rangle\right)$. We denote the expression of the edge states as

$$
\left|\psi_{L, R}\right\rangle=\left(\psi_{1 A}, \psi_{1 B}, \ldots, \psi_{N A}, \psi_{N B}\right)
$$

where $N$ is the total number of the unit cells. The edge states at the limitation of infinity large size system $(N \rightarrow \infty)$ are analytically obtained. For the edge state $\left|\psi_{L}\right\rangle$, the components of $\left|\psi_{L}\right\rangle$ in the $n$th unit cell are $\left(\psi_{n A}, \psi_{n B}\right)=\left(\rho^{n-1}, 0\right)$ with the decay factor $\rho=-\left(\lambda+2 \cos k_{y}\right) / \kappa$. For the edge state $\left|\psi_{R}\right\rangle$, the components of $\left|\psi_{R}\right\rangle$ in the $n$th unit cell are $\left(\psi_{n A}, \psi_{n B}\right)=$ $\left(0, \rho^{N-n}\right)$. The expression is valid for all the three types of topological edge states.

\section{TOPOLOGICAL CHARACTERIZATION}

Nonspatial (internal) symmetry plays a key role in the topological classification and determines the topological

characterization [11]. Time-reversal symmetry (TRS), particle-hole symmetry (PHS), and chiral symmetry (CS) classify the topological phases in a tenfold way [75]. The time-reversal symmetry is defined as

$$
\text { TRS : } \quad \mathcal{T} h(\mathbf{k}) \mathcal{T}^{-1}=h(-\mathbf{k}), \quad \mathcal{T}^{2}= \pm 1
$$

The time-reversal operator $\mathcal{T}=U K$ is an anti-unitary operator, where $U$ is a linear operator and the complex conjugation $K$ is an antilinear operator.

The TRS ensures that the Berry curvature $F$ satisfies $F(\mathbf{k})=-F(-\mathbf{k})$, the integral of $F$ in the entire BZ results in a vanishing Chern number. How to characterize the band topology and determine the topologically nontrivial phase of a time-reversal symmetric system? For the timereversal symmetric system with $\mathcal{T}^{2}=-1$, the topological properties can be characterized by a $Z_{2}$ topological invariant. $Z_{2}=0$ represents the trivial insulator and $Z_{2}=1$ represents the topological insulator. The Pfaffian method, time-reversal polarization, and non-Abelian Berry connection have been developed as the $Z_{2}$ invariant, but these are unable to describe the topological systems with $\mathcal{T}^{2}=+1$. For the time-reversal symmetric system with $\mathcal{T}^{2}=+1$, the $2 \mathrm{D}$ Zak phase, also known as the wave polarization, is used for the topological characterization. The wave polarization is quantized to 0 or $1 / 2$ under the symmetry protection and is used to describe the band topology of the time-reversal symmetric system with $\mathcal{T}^{2}=+1$ [45]. The topological edge states appear inside the band gap [74].

For the Bloch Hamiltonian of the 2D square lattice $h(\mathbf{k})$, the linear operator $U=\sigma_{0}$ is the $2 \times 2$ identical matrix; thus, the $2 \mathrm{D}$ square lattice possesses the time-reversal symmetry of $\mathcal{T}^{2}=+1$. The term $d_{0} \sigma_{0}$ in the Bloch Hamiltonian $h(\mathbf{k})$ is crucial for the appearance of the metallic phase; however, $d_{0} \sigma_{0}$ only adjusts the band energy, but not the eigenstates of $h(\mathbf{k})$. The topology of $h(\mathbf{k})$ only relates to the eigenstate and thus is determined from $\mathbf{d} \cdot \sigma$. The eigenstate of $h(\mathbf{k})$ is in the form of

$$
\left|\Psi_{ \pm}(\mathbf{k})\right\rangle=\frac{1}{\Omega_{ \pm}}\left(\begin{array}{c}
d_{z} \pm d \\
d_{x}+i d_{y}
\end{array}\right),
$$

where the subscript + represents the upper band and the subscript - represents the lower band; and $\Omega_{ \pm}=\sqrt{2 d\left(d \pm d_{z}\right)}$ is the normalization factor.

The wave polarization is defined as

$$
\mathbf{P}=\frac{1}{2 \pi} \int_{B Z} \operatorname{Tr}\left[\mathbf{A}\left(k_{x}, k_{y}\right)\right] d k_{x} d k_{y},
$$

where $\mathbf{A}\left(k_{x}, k_{y}\right)=\left\langle\Psi(\mathbf{k})\left|i \nabla_{\mathbf{k}}\right| \Psi(\mathbf{k})\right\rangle$ is the Berry connection. $\mathbf{P}=\left(P_{x}, P_{y}\right)$ includes two components, where $P_{x}$ characterizes the topology in the $x$ direction and predicts the edge states for the 2D square lattice under the $\mathrm{OBC}$ in the $x$ direction. $P_{y}$ characterizes the topology in the $y$ direction. The wave polarization defines the 2D Zak phase $\Theta_{v}=2 \pi P_{v}(v=x, y)$. Protected by the reflection symmetry or inversion symmetry, the value of wave polarization is quantized, being 0 or $\pm 1 / 2$. For the Bloch Hamiltonian $h(\mathbf{k})$, the reflection symmetry in the $y$ direction guarantees $P_{y}=0$. The Bloch Hamiltonian $h(\mathbf{k})$ is reflection symmetric in the $y$ direction $h\left(k_{x}, k_{y}\right)=$ $h\left(k_{x},-k_{y}\right) . P_{y}=0$ reflects the lack of nontrivial edge state for 


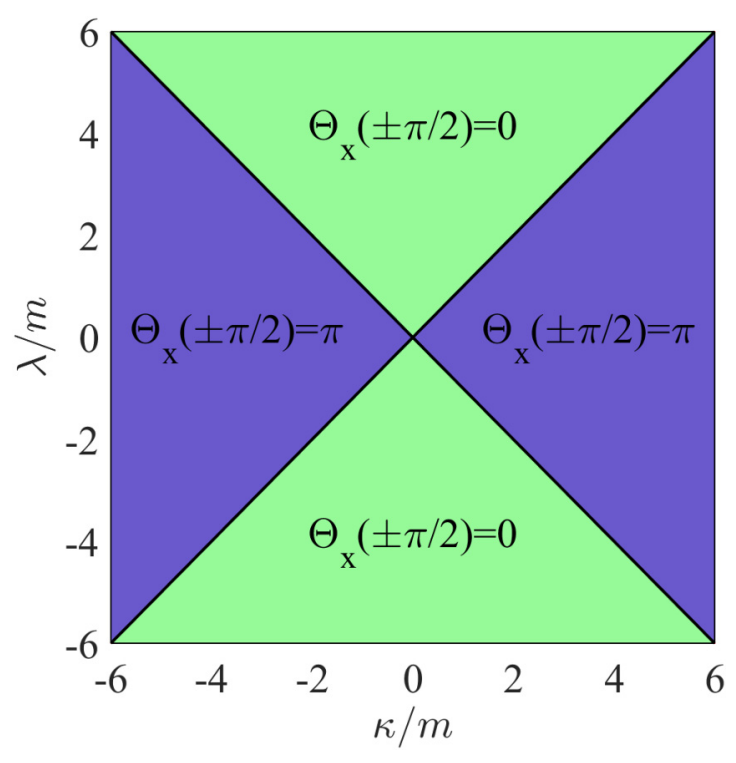

FIG. 6. Topological phase diagram of the time-reversal symmetric 2D square lattice. The $|\lambda|<|\kappa|$ region in purple is the topologically nontrivial phase; in contrast, the $|\lambda|>|\kappa|$ region in green is the trivial phase. The Zak phase $\Theta_{x}$ distinguishes different topological phases.

the 2D square lattice under the $\mathrm{OBC}$ in the $y$ direction and the $\mathrm{PBC}$ in the $x$ direction.

The polarization $P_{x}$ is not quantized without the symmetry protection. Therefore, $P_{x}$ is invalid for the topological characterization. We apply a Fourier transformation to decompose the 2D lattice Hamiltonian into a decoupled 1D lattice Hamiltonian parametrized by the momentum $k_{y}$. The band gap closes at $k_{y}= \pm \pi / 2$, where the topological phase transition occurs and the edge states intersect. For $\lambda=-\kappa(\kappa \neq 0)$, the band degenerate points locate at $\left(k_{x}, k_{y}\right)=(0, \pm \pi / 2)$. For $\lambda=\kappa(|\kappa| \leqslant 1)$, the band gap closes at $\left(k_{x}, k_{y}\right)=$ $(0, \pm \arccos \lambda)$ and $(\pi, \pm \pi / 2)$. For $\lambda=\kappa(|\kappa|>1)$, the band degenerate points locate at $\left(k_{x}, k_{y}\right)=(\pi, \pm \pi / 2)$. In particular, when $(\lambda, \kappa)=(0,0)$, the band degenerate points become two degenerate lines at $k_{y}= \pm \pi / 2$.

The 1D Zak phase is defined as

$$
\Theta_{x}\left(k_{y}\right)=\int\left\langle\psi\left(k_{x}\right)\left|i \partial_{k_{x}}\right| \psi\left(k_{x}\right)\right\rangle d k_{x}
$$

which reflects the winding of the Bloch Hamiltonian when $k_{x}$ varies throughout the entire $\mathrm{BZ}$ of the decoupled 1D lattice. The 1D Zak phase $\Theta_{x}( \pm \pi / 2)$ is quantized to $\pi$ in the topologically nontrivial phases (the purple and cyan regions in Fig. 2), whereas $\Theta_{x}( \pm \pi / 2)$ is quantized to 0 in the trivial phase (the green and yellow regions in Fig. 2). The topological characterization is shown in Fig. 6 and is consistent with the phase diagram in Fig. 2. $\Theta_{x}( \pm \pi / 2)=\pi$ characterizes the topological metal and topological insulator. $\Theta_{x}( \pm \pi / 2)=0$ characterizes the ordinary metal and ordinary insulator.

\section{DISCUSSION}

The vertical coupling $\lambda \neq \pm \kappa$ is crucial for the topological metallic phase as indicated in Fig. 2. In addition, we consider the horizontal couplings $\lambda$ and $\kappa$ are replaced by $\lambda^{\prime}$ and $\kappa^{\prime}$. Then, when $\lambda^{\prime}=\kappa^{\prime}$, the energy band must have degenerate points located at $\left(k_{x}, k_{y}\right)=( \pm \pi, \pm \pi / 2)$; when $\lambda^{\prime}=-\kappa^{\prime}$, the energy band must have degenerate points located at $\left(k_{x}, k_{y}\right)=$ $(0, \pm \pi / 2)$. Notably, $\lambda^{\prime} \neq \pm \kappa^{\prime}$ is necessary for the topological metallic phase, but $\lambda=\lambda^{\prime}$ and $\kappa=\kappa^{\prime}$ are not necessary. The nearest neighbor coupling $m \neq 0$ is important for the different edge states in the topological metallic phases. For $m=0$, only the in-gap edge states exist in the topological phase and only one type of topological phase presents in this situation.

The spin-orbit coupling is used to create the magnetic flux and the traditional topological metal. However, the proposed 2D topological metal is time-reversal symmetric without the magnetic flux. This requires the spin-orbit coupling is negligible in the material. The organic materials composed of light elements have weak spin-orbit coupling [78]. Therefore, the organic materials may be suitable candidates for the class AI topological phase [79].

If the time-reversal symmetry breaks when the square lattice is threaded by the magnetic flux. The topological metallic phase still exists through tuning the magnetic flux and the coupling strengths. In this situation, the topological phase is characterized by the nonzero Chern number. Under the PBC in the $y$ direction, a pair of antichiral edge states exist after imposing the $\mathrm{OBC}$ in the $x$ direction. The antichiral edge states also connect the upper and lower energy bands to form the conductive surface states, but the two antichiral edge states on different boundaries propagate in the same direction in contrast to the chiral edge states [80].

Furthermore, the non-Hermiticity compresses the band gap between the valence band and the conduction band. The nonHermitian topological metallic phase exits in the proposed 2D square lattice when the additional gain and loss are presented in different sublattices. This is an interesting topic that deserving further investigation.

\section{CONCLUSION}

In conclusion, we provide an alternative way of generating the topological metals. The proposed topological metallic phases are protected by the time-reversal symmetry and are free from magnetic flux. The time-reversal symmetry ensures a zero Chern number; however, the 2D square lattice supports topologically nontrivial phases, which are distinguished by the different types of edge states. The 2D square lattice supports three different cases in the topological metallic phase that significantly differ from the traditional topological metallic phases created through tuning the magnetic flux. These different cases can be deformed into each other without topological phase transition. Our findings provide insights into the time-reversal symmetric topological metals.

\section{ACKNOWLEDGMENTS}

This work was supported by the National Natural Science Foundation of China (Grants No. 11975128 and No. $11874225)$ 
[1] L. Fu, C. L. Kane, and E. J. Mele, Topological Insulators in Three Dimensions, Phys. Rev. Lett. 98, 106803 (2007).

[2] M. Z. Hasan and C. L. Kane, Colloquium: Topological insulators, Rev. Mod. Phys. 82, 3045 (2010).

[3] X.-G. Wen, Colloquium: Zoo of quantum-topological phases of matter, Rev. Mod. Phys. 89, 041004 (2017).

[4] A. Bansil, H. Lin, and T. Das, Colloquium: Topological band theory, Rev. Mod. Phys. 88, 021004 (2016).

[5] X.-L. Qi and S.-C. Zhang, Topological insulators and superconductors, Rev. Mod. Phys. 83, 1057 (2011).

[6] A. A. Burkov and L. Balents, Weyl Semimetal in a Topological Insulator Multilayer, Phys. Rev. Lett. 107, 127205 (2011).

[7] S. M. Young, S. Zaheer, J. C. Y. Teo, C. L. Kane, E. J. Mele, and A. M. Rappe, Dirac Semimetal in Three Dimensions, Phys. Rev. Lett. 108, 140405 (2012).

[8] N. P. Armitage, E. J. Mele, and A. Vishwanath, Weyl and Dirac semimetals in three-dimensional solids, Rev. Mod. Phys. 90, 015001 (2018).

[9] A. A. Burkov, Topological semimetals, Nat. Mater. 15, 1145 (2016).

[10] B. Yan and C. Felser, Topological materials: Weyl semimetals, Annu. Rev. Condens. Matter Phys. 8, 337 (2017).

[11] C. K. Chiu, J. C. Teo, A. P. Schnyder, and S. Ryu, Classification of topological quantum matter with symmetries, Rev. Mod. Phys. 88, 035005 (2016).

[12] L. Jin and Z. Song, Bulk-boundary correspondence in a nonHermitian system in one dimension with chiral inversion symmetry, Phys. Rev. B 99, 081103(R) (2019).

[13] K. Kawabata, K. Shiozaki, M. Ueda, and M. Sato, Symmetry and Topology in Non-Hermitian Physics, Phys. Rev. X 9, 041015 (2019).

[14] Y. Ashida, Z. Gong, and M. Ueda, Non-Hermitian physics, Adv. Phys. 69, 249 (2020).

[15] D. J. Thouless, M. Kohmoto, M. P. Nightingale, and M. den Nijs, Quantized Hall Conductance in a Two-Dimensional Periodic Potential, Phys. Rev. Lett. 49, 405 (1982).

[16] D. Xiao, M.-C. Chang, and Q. Niu, Berry phase effects on electronic properties, Rev. Mod. Phys. 82, 1959 (2010).

[17] J. C. Y. Teo and C. L. Kane, Topological defects and gapless modes in insulators and superconductors, Phys. Rev. B 82, 115120 (2010).

[18] Y. Ran, Y. Zhang, and A. Vishwanath, One-dimensional topologically protected modes in topological insulators with lattice dislocations, Nat. Phys. 5, 298 (2009).

[19] L. J. Lang, X. Cai, and S. Chen, Edge States and Topological Phases in One-Dimensional Optical Superlattices, Phys. Rev. Lett. 108, 220401 (2012).

[20] X.-J. Liu, K. T. Law, T. K. Ng, and P. A. Lee, Detecting Topological Phases in Cold Atoms, Phys. Rev. Lett. 111, 120402 (2013).

[21] M. Aidelsburger, M. Atala, M. Lohse, J. T. Barreiro, B. Paredes, and I. Bloch, Realization of the Hofstadter Hamiltonian with Ultracold Atoms in Optical Lattices, Phys. Rev. Lett. 111, 185301 (2013).

[22] L. Lu, J. D. Joannopoulos, and M. Soljačić, Topological photonics, Nat. Photon. 8, 821 (2014).

[23] S.-Y. Yu, C. He, Z. Wang, F.-K. Liu, X.-C. Sun, Z. Li, H.-Z. Lu, M.-H. Lu, X.-P. Liu, and Y.-F. Chen, Elastic pseudospin transport for integratable topological phononic circuits, Nat. Commun. 9, 3072 (2018).
[24] Y.-B. Yang, L.-M. Duan, and Y. Xu, Continuously tunable topological pump in high-dimensional cold atomic gases, Phys. Rev. B 98, 165128 (2018).

[25] D.-W. Zhang, Y.-Q. Zhu, Y. X. Zhao, H. Yan, and S.-L. Zhu, Topological quantum matter with cold atoms, Adv. Phys. 67, 253 (2019).

[26] X.-D. Chen, W.-M. Deng, F.-L. Shi, F.-L. Zhao, M. Chen, and J.-W. Dong, Direct Observation of Corner States in SecondOrder Topological Photonic Crystal Slabs, Phys. Rev. Lett. 122, 233902 (2019).

[27] Y. Xu, Topological gapless matters in three-dimensional ultracold atomic gases, Front. Phys. 14, 43402 (2019).

[28] S. Imhof, C. Berger, F. Bayer, J. Brehm, L. Molenkamp, T. Kiessling, F. Schindler, C. H. Lee, M. Greiter, T. Neupert, and R. Thomale, Topolectrical-circuit realization of topological corner modes, Nat. Phys. 14, 925 (2018).

[29] T. Helbig, T. Hofmann, C. H. Lee, R. Thomale, S. Imhof, L. W. Molenkamp, and T. Kiessling, Band structure engineering and reconstruction in electric circuit networks, Phys. Rev. B 99, 161114(R) (2019).

[30] Y. Lu, N. Jia, L. Su, C. Owens, G. Juzeliunas, D. I. Schuster, and J. Simon, Probing the Berry curvature and Fermi arcs of a Weyl circuit, Phys. Rev. B 99, 020302(R) (2019).

[31] S. Murakami, N. Nagaosa, and S. C. Zhang, Spin-Hall Insulator, Phys. Rev. Lett. 93, 156804 (2004).

[32] C. L. Kane and E. J. Mele, Quantum Spin Hall Effect in Graphene, Phys. Rev. Lett. 95, 226801 (2005).

[33] B. A. Bernevig, T. L. Hughes, and S. C. Zhang, Quantum spin Hall effect and topological phase transition in $\mathrm{HgTe}$ quantum wells, Science 314, 1757 (2006).

[34] X.-L. Qi, Y.-S. Wu, and S.-C. Zhang, Topological quantization of the spin Hall effect in two-dimensional paramagnetic semiconductors, Phys. Rev. B 74, 085308 (2006).

[35] C. L. Kane and E. J. Mele, $\mathbb{Z}_{2}$ Topological Order and the Quantum Spin Hall Effect, Phys. Rev. Lett. 95, 146802 (2005).

[36] A. Roth, C. Brüne, H. Buhmann, L. W. Molenkamp, J. Maciejko, X. L. Qi, and S. C. Zhang, Nonlocal transport in the quantum spin Hall state, Science 325, 294 (2009).

[37] L. Sheng, D. N. Sheng, C. S. Ting, and F. D. M. Haldane, Nondissipative spin Hall Effect via Quantized Edge Transport, Phys. Rev. Lett. 95, 136602 (2005).

[38] E. Prodan, Robustness of the spin-Chern number, Phys. Rev. B 80, 125327 (2009).

[39] Y. Yang, Z. Xu, L. Sheng, B. Wang, D. Y. Xing, and D. N. Sheng, Time-Reversal-Symmetry-Broken Quantum Spin Hall Effect, Phys. Rev. Lett. 107, 066602 (2011).

[40] F. Zhang, J. Jung, G. A. Fiete, Q. Niu, and A. H. MacDonald, Spontaneous Quantum Hall States in Chirally Stacked FewLayer Graphene Systems, Phys. Rev. Lett. 106, 156801 (2011).

[41] F. Zhang, A. H. MacDonald, and E. J. Mele, Valley Chern numbers and boundary modes in gapped bilayer graphene, Proc. Natl. Acad. Sci. U.S.A. 110, 10546 (2013).

[42] M. Ezawa, Topological Kirchhoff law and bulk-edge correspondence for valley Chern and spin-valley Chern numbers, Phys. Rev. B 88, 161406(R) (2013).

[43] R. Resta, Macroscopic polarization in crystalline dielectrics: The geometric phase approach, Rev. Mod. Phys. 66, 899 (1994).

[44] P. Delplace, D. Ullmo, and G. Montambaux, The Zak phase and the existence of edge states in graphene, Phys. Rev. B 84, 195452 (2011). 
[45] F. Liu and K. Wakabayashi, Novel Topological Phase with a Zero Berry Curvature, Phys. Rev. Lett. 118, 076803 (2017).

[46] S. Murakami, Phase transition between the quantum spin Hall and insulator phases in 3D: Emergence of a topological gapless phase, New J. Phys. 9, 356 (2007).

[47] X. Wan, A. M. Turner, A. Vishwanath, and S. Y. Savrasov, Topological semimetal and Fermi-arc surface states in the electronic structure of pyrochloreiridates, Phys. Rev. B 83, 205101 (2011).

[48] Z. Wang, H. Weng, Q. Wu, X. Dai, and Z. Fang, Threedimensional Dirac semimetal and quantum transport in $\mathrm{Cd}_{3} \mathrm{As}_{2}$, Phys. Rev. B 88, 125427 (2013).

[49] Z. Zhu, G. W. Winkler, Q. Wu, J. Li, and A. A. Soluyanov, Triple Point Topological Metals, Phys. Rev. X 6, 031003 (2016).

[50] B. J. Wieder, Y. Kim, A. Rappe, and C. Kane, Double Dirac Semimetals in Three Dimensions, Phys. Rev. Lett. 116, 186402 (2016).

[51] H. Weng, C. Fang, Z. Fang, and X. Dai, Topological semimetals with triply degenerate nodal points in $\theta$-phase tantalum nitride, Phys. Rev. B 93, 241202(R) (2016).

[52] S. Singh, Q. Wu, C. Yue, A. H. Romero, and A. A. Soluyanov, Topological phonons and thermoelectricity in triple-point metals, Phys. Rev. Materials 2, 114204 (2018).

[53] G. Shan and H. B. Gao, New topological semimetal candidate of nonsymmorphic $\mathrm{PdSb}_{2}$ with unique six-fold degenerate point, Front. Phys. 14, 43201 (2019).

[54] R. Chapai, Y. Jia, W. Shelton, R. Nepal, M. Saghayezhian, J. DiTusa, E. Plummer, C. Jin, and R. Jin, Fermions and bosons in nonsymmorphic $\mathrm{PdSb}_{2}$ with six-fold degeneracy, Phys. Rev. B 99, 161110(R) (2019).

[55] A. A. Burkov, M. D. Hook, and L. Balents, Topological nodal semimetals, Phys. Rev. B 84, 235126 (2011).

[56] R. Yu, H. M. Weng, Z. Fang, X. Dai, and X. Hu, Topological Node-line Semimetal and Dirac Semimetal State in Antiperovskite $\mathrm{Cu}_{3}$ PdN, Phys. Rev. Lett. 115, 036807 (2015).

[57] H. M. Weng, Y. Y. Liang, Q. N. Xu, R. Yu, Z. Fang, X. Dai, and Y. Kawazoe, Topological node-line semimetal in three-dimensional graphene networks, Phys. Rev. B 92, 045108 (2015).

[58] W. Wu, Y. Liu, S. Li, C. Zhong, Z.-M. Yu, X.-L. Sheng, Y. X. Zhao, and S. A. Yang, Nodal surface semimetals: Theory and material realization, Phys. Rev. B 97, 115125 (2018).

[59] A. A. Soluyanov, D. Gresch, Z. Wang, Q. Wu, M. Troyer, X. Dai, and B. A. Bernevig, Type-II Weyl semimetals, Nature (London) 527, 495 (2015).

[60] Y. Xu, F. Zhang, and C. Zhang, Structured Weyl Points in Spin-Orbit Coupled Fermionic Superfluids, Phys. Rev. Lett. 115, 265304 (2015).

[61] S. Li, Z. M. Yu, Y. Liu, S. Guan, S. S. Wang, X. Zhang, Y. Yao, and S. A. Yang, Type-II nodal loops: Theory and material realization, Phys. Rev. B 96, 081106(R) (2017).

[62] M. Bahari and M. V. Hosseini, One-dimensional topological metal, Phys. Rev. B 99, 155128 (2019).
[63] S.-Y. Xu, C. Liu, S. K. Kushwaha, R. Sankar, J. W. Krizan, I. Belopolski, M. Neupane, G. Bian, N. Alidoust, T.-R. Chang et al., Observation of Fermi arc surface states in a topological metal: A new type of 2D electron gas, Science 347, 294 (2015).

[64] Z. Wang, D. Gresch, A. A. Soluyanov, W. Xie, S. Kushwaha X. Dai, M. Troyer, R. J. Cava, and B. A. Bernevig, $\mathrm{MoTe}_{2}$ : A Type-II Weyl Topological Metal, Phys. Rev. Lett. 117, 056805 (2016).

[65] X. Z. Ying and A. Kamenev, Symmetry-Protected Topological Metals, Phys. Rev. Lett. 121, 086810 (2018).

[66] M. Aidelsburger, M. Lohse, C. Schweizer, M. Atala, J. T. Barreiro, S. Nascimbène, N. R. Cooper, I. Bloch, and N. Goldman, Measuring the Chern number of Hofstadter bands with ultracold bosonic atom, Nat. Phys. 11, 162 (2015).

[67] S. Mittal, E. A. Goldschmidt, and M. Hafezi, A topological source of quantum light, Nature (London) 561, 502 (2018).

[68] T. Hofmann, T. Helbig, C. H. Lee, M. Greiter, and R. Thomale, Chiral Voltage Propagation and Calibration in a Topolectrical Chern Circuit, Phys. Rev. Lett. 122, 247702 (2019).

[69] Z. J. Yang, F. Gao, X. H. Shi, X. Lin, Z. Gao, Y. D. Chong, and B. L. Zhang, Topological Acoustics, Phys. Rev. Lett. 114, 114301 (2015).

[70] C. He, X. Ni, H. Ge, X.-C. Sun, Y.-B. Chen, M.-H. Lu, X.-P. Liu, and Y.-F. Chen, Acoustic topological insulator and robust one-way sound transport, Nat. Phys. 12, 1124 (2016).

[71] Y. G. Peng, C. Z. Qin, D. G. Zhao, Y. X. Shen, X. Y. Xu, M. Bao, H. Jia, and X. F. Zhu, Experimental demonstration of anomalous Floquet topological insulator for sound, Nat. Commun. 7, 13368 (2016).

[72] J. Y. Lu, C. Y. Qiu, W. Y. Deng, X. Q. Huang, F. Li, F. Zhang, S. Q. Chen, and Z. Y. Liu, Valley Topological Phases in Bilayer Sonic Crystals, Phys. Rev. Lett. 120, 116802 (2018).

[73] L. Jin and Z. Song, Symmetry-protected scattering in nonHermitian linear systems, Chin. Phys. Lett. 38, 024202 (2021).

[74] H. C. Wu, L. Jin, and Z. Song, Nontrivial topological phase with a zero Chern number, Phys. Rev. B 102, 035145 (2020).

[75] S. Ryu, A. P. Schnyder, A. Furusaki, and A. W. W. Ludwig, Topological insulators and superconductors: tenfold way and dimensional hierarchy, New J. Phys 12, 065010 (2010).

[76] X. Wen, C. Qiu, Y. Qi, L. Ye, M. Ke, F. Zhang, and Z. Liu, Acoustic Landau quantization and quantum-Hall-like edge states, Nat. Phys. 15, 352 (2019).

[77] N. H. Le, A. J. Fisher, N. J. Curson, and E. Ginossar, Topological phases of a dimerized Fermi-Hubbard model for semiconductor nano-lattices, npj Quantum Inf. 6, 24 (2020).

[78] L. Jin, M. Pfender, N. Aslam, P. Neumann, S. Yang, J. Wrachtrup, and R.-B. Liu, Proposal for a room-temperature diamond maser, Nat. Commun. 6, 8251 (2015).

[79] Z. S. Liao, H. H. Zhang, and Z. B. Yan, Nonlinear hall effect in two-dimensional class AI metals, Phys. Rev. B 103, 235151 (2021).

[80] E. Colomés and M. Franz, Antichiral Edge States in a Modified Haldane Nanoribbon, Phys. Rev. Lett. 120, 086603 (2018). 